\title{
miR-21 and its target gene CCL20 are both highly overexpressed in the microenvironment of colorectal tumors: Significance of their regulation
}

\author{
BENJAMIN VICINUS ${ }^{1 *}$, CLAUDIA RUBIE ${ }^{1 *}$, NATHALIE STEGMAIER ${ }^{1}$, VILMA OLIVEIRA FRICK ${ }^{1}$, \\ KATHRIN KÖLSCH ${ }^{1}$, ANNE KAUFFELS ${ }^{1}$, PIRUS GHADJAR ${ }^{2}$, \\ MATHIAS WAGNER $^{3}$ and MATTHIAS GLANEMANN ${ }^{1}$
}

\author{
${ }^{1}$ Department of General, Visceral, Vascular and Paediatric Surgery, University of the Saarland, 66421 Homburg/Saar, \\ Germany; ${ }^{2}$ Department of Radiation Oncology, Inselspital, Bern University Hospital and University of Bern, 3010 Bern, \\ Switzerland; ${ }^{3}$ Institute of Pathology, University of the Saarland, 66421 Homburg/Saar, Germany
}

Received March 19, 2013; Accepted June 12, 2013

DOI: $10.3892 /$ or.2013.2580

\begin{abstract}
Recently, we reported a functional interaction between miR-21 and its identified chemokine target CCL20 in colorectal cancer (CRC) cell lines. Here, we investigated whether such functional interactions are permitted at the cellular level which would require an inverse correlation of expression and also co-expression of miR-21 and CCL20 in the same cell. Expression profiling was performed using qPCR, and ELISA, in situ hybridization and immunohistochemistry were applied for the presentation of their cellular localization. We demonstrated that miR-21 as well as CCL20 were both significantly upregulated in CRC tissues; thus, showing no antidromic expression pattern. This provided an initial clue that miR-21 and CCL20 may not be expressed in the same cell. In addition, we located miR-21 expression at the cellular level predominantly in stromal cells such as tumor-associated fibroblasts and to a minor degree in immune cells such as macrophages and lymphocytes. Likewise, CCL20 expression was primarily detected in tumor-infiltrating immune cells. Thus, investigating the cellular localization of miR-21 and its target CCL20 revealed that both molecules are expressed predominantly in the microenvironment of CRC tumors.
\end{abstract}

\section{Introduction}

microRNAs (miRNAs) are small 22-nt long, endogenous, non-coding RNA molecules which are involved in the post-

Correspondence to: Dr Claudia Rubie, Department of General, Visceral, Vascular and Paediatric Surgery, University of the Saarland, Kirrberger Strasse, 66421 Homburg/Saar, Germany

E-mail: ca.labor@uks.eu

${ }^{*}$ Contributed equally

Key words: miR-21, CCL20, colorectal carcinoma, gene expression, cellular localization transcriptional regulation of gene expression (1). They mediate their regulatory function through interactions with the RNA-induced silencing complex (RISC) which is guided to target messenger-RNAs (mRNAs) by sequences which are partially complementary to the seed region of the loaded miRNAs $(1,2)$. The targeted mRNA can be affected by the miRNA-RISC complex in two ways: (i) inhibition of translation or (ii) degradation of mRNA (3). It was reported that miRNAs regulate the expression of $\sim 30 \%$ of all human proteins (4) and therefore miRNAs are proposed to have a huge impact on post-transcriptional gene regulation.

miRNAs are transcribed in the nucleus by RNA polymerase II or III resulting in so-called primary-miRNAs (pri-miRNA) $(5,6)$. These pri-miRNAs are further processed to $\sim 60-70$ bp long hairpin precursor (pre-miRNA) through nuclear cleavage by a protein complex containing the RNase III endonuclease Drosha (7). The processed pre-miRNAs are exported from the nucleus to the cytoplasm by Exportin-5 (8) where they are further processed by Dicer resulting in mature miRNAs. Alterations in any of the processing steps or epigenetic modification of miRNA genes potentially result in deficient miRNA expression.

Since the discovery of miRNAs, deficient miRNA expression moved into the focus of investigation related to the development and progression of cancer. In recent years, dysregulated expression of miRNAs was detected as a crucial factor and prognostic feature of various cancer entities. However, deficient miRNA expression has the potential to influence the expression of both proto-oncogenes and tumorsuppressor genes in a direct or indirect manner (9). Thus, the impact of dysregulated miRNAs on cancer depends on the subset of target genes regulated by the particular miRNA. One example of a proto-oncogenic effect of a dysregulated miRNA is represented by overexpression of miR-152 in endometrial cancer cell lines, which results in decreased growth of tumor cells (10). In contrast, inhibition of miR-21 in breast cancer cells decreases proliferation, migration and tumor growth (11). In this respect, miRNAs that downregulate tumor-suppressor genes are designated as oncomiRs 
whereas miRNAs that control oncogenes are designated as tumor-suppressor miRNAs. In colorectal carcinoma (CRC), various miRNAs have been identified which were shown to be dysregulated and exhibit upregulation or downregulation compared to corresponding unaffected tissues $(12,13)$. In this respect, miR-21 was proposed to act as an oncomiR in CRC (14).

Recently, various miRNAs were shown to target miscellaneous chemokines in different cancer entities $(15,16)$. In this respect, chemokine (C-C-motif) 20 (CCL20) was shown to play a role in CRC progression and metastasis via its interaction with its receptor CCR6 $(17,18)$. Recently, we reported a functional interaction between miR-21 and its identified target gene CCL20 in CRC cells (16). However, for functional interaction of miRNAs with their target genes, both molecules must be present in the same cell.

Since dysregulated miRNA expression has been observed in tumor cells (19) as well as in the microenvironment of tumor (20), the cellular localization of miR-21 and its target gene CCL20 has moved into the focus of our interest. Hence, the present study aimed to investigate the expression profile and cellular distribution of miR-21 and its target gene CCL20 in tissue specimens from CRC patients.

\section{Materials and methods}

Patients and samples. Surgical specimens and corresponding normal tissue from the same samples were collected from patients who underwent surgical resection at our department between 2003 and 2010. No patient underwent any specific cancer therapy prior to the resection. A total of 46 patients with different stages of CRC were analyzed. The patient characteristics and clinical data were obtained from a prospective database and are summarized in Table I. Informed consent for tissue procurement was obtained from all patients. The study was approved by the Ethics Commission of the Ärztekammer of the Saarland.

Tissue preparation. All tissue specimens were snap frozen in liquid nitrogen directly after surgical resection and stored at $-80^{\circ} \mathrm{C}$ until further processing. The steps were performed under nucleic acid sterile conditions. Regarding corresponding normal tissue, adjacent non-affected tissue from the same resection specimen was applied. All tissues were evaluated by an experienced pathologist, and only CRC tissues comprising at least $70 \%$ tumor area were applied.

Single-strand cDNA synthesis. The mirVana miRNA isolation kit (Life Technologies, Carlsbad, CA, USA) was used to isolate total RNA from the tissue samples according to the manufacturer's protocol. The quality of RNA was checked spectrophotometrically and by electrophoresis on $1 \%$ agarose gels.

For synthesis of cDNA, $1 \mu \mathrm{g}$ of each tissue total RNA was reverse transcribed in a final reaction volume of $50 \mu \mathrm{l}$. Each reaction contained $1 \mathrm{X}$ TaqMan RT buffer, $2.5 \mu \mathrm{M}$ random hexamers, $500 \mu \mathrm{M}$ each dNTP, $5.5 \mathrm{mM} \mathrm{MgCl}_{2}, 0.4 \mathrm{U} / \mu \mathrm{l}$ RNase inhibitor, and $1.25 \mathrm{U} / \mu 1$ MultiScribe RT. The cycler program was $10 \mathrm{~min}$ at $25^{\circ} \mathrm{C}, 90 \mathrm{~min}$ at $48^{\circ} \mathrm{C}$, and $5 \mathrm{~min}$ at $95^{\circ} \mathrm{C}$. For reverse transcription of miRNAs, $10 \mathrm{ng}$ of each total
Table I. Clinical characteristics of the patients with colorectal carcinoma.

\begin{tabular}{lc}
\hline Characteristic & $\begin{array}{c}\text { No. of } \\
\text { patients }(n=46)\end{array}$ \\
\hline
\end{tabular}

Localization of primary tumor

Colon $\quad 23$

Rectum 23

Gender

Male $\quad 28$

Female 18

Age at surgery (years)

Median $\quad 68.3$

Range 44-88

Tumor (T)-classification of the primary tumor

$\mathrm{T} 1$

$\mathrm{T} 2$

12

T3

16

T4

Lymph node status

Positive

Negative

AJCC stage of the primary tumor I

II

III

IV

Grade

G1 4

G2 19

G3 21

G4

2

RNA sample was attached in a final reaction volume of $15 \mu \mathrm{l}$. Each reaction included $1 \mathrm{X}$ RT buffer, $3 \mu \mathrm{l}$ small RNA-specific RT primer, $1 \mathrm{mM}$ dNTPs, $0.25 \mathrm{U} / \mu 1$ RNase inhibitor, and $3.33 \mathrm{U} / \mu 1$ MultiScribe RT. The cycler program was $30 \mathrm{~min}$ at $16^{\circ} \mathrm{C}, 30 \mathrm{~min}$ at $42^{\circ} \mathrm{C}$ and $5 \mathrm{~min}$ at $85^{\circ} \mathrm{C}$. All RT-PCR reagents were purchased from Life Technologies and used according to the manufacturer's instructions.

Quantitative real-time PCR. CCL20 mRNA detection was performed in a q-RT-PCR reaction containing $10 \mu \mathrm{l}$ 2X TaqMan Universal PCR Master Mix II and $1 \mu$ l CCL20 gene expression assay (Life Technologies), $8 \mu$ l RNase-free water and $1 \mu \mathrm{l}$ cDNA template $(20 \mathrm{ng} / \mu \mathrm{l})$. miRNA quantification assays contained $10 \mu \mathrm{l}$ 2X TaqMan Universal PCR Master Mix II and $1 \mu \mathrm{l}$ 20X TaqMan Small RNA assay (Life Technologies), $7.67 \mu 1$ RNase-free water and $1.33 \mu \mathrm{l}$ cDNA template $(0.66 \mathrm{ng} / \mu \mathrm{l})$. The theoretical basis of q-RT assays is described in detail elsewhere (21). Reactions were run in the ABI Prism 7900 sequence detector (Life Technologies) with an initial step of $10 \mathrm{~min}$ at $95^{\circ} \mathrm{C}$, followed by 40 thermal cycles 
of $15 \mathrm{sec}$ at $95^{\circ} \mathrm{C}$ and $10 \mathrm{~min}$ at $60^{\circ} \mathrm{C}$ and the log-linear phase of amplification was monitored to obtain $\mathrm{Ct}$ values for each RNA sample. All reactions were run in triplicates together with a no template control and a DNA contamination control where the reverse transcriptase was omitted. CCL20 mRNA (RefSeq: NM_004591.1) expression was normalized according to the expression of the slope matched housekeeping gene $\beta$-2-microglobulin (b2m) (RefSeq: NM_004048.2) applying the $2^{-\Delta \Delta C_{t}}$ method (22).

Fold difference $=2^{-\left(\text {mean } C_{t} \text { pathological tissue - mean } C_{t} \text { calibrator }\right)}=2^{-\Delta C_{t}}$

Expression of miR-21 (miRBase: MI0000077) was analyzed applying the $2^{-\Delta \Delta \mathrm{C}_{\mathrm{t}}}$ method relative to the levels of RNU48 (RefSeq: NR_002745.1) using a special miR-21 detection assay (assay ID, 000397; Life Technologies) for q-RT-PCR. miR-21 expression data are depicted as n-fold expression relative to the corresponding normal colorectal tissues.

Isolation of total protein. Protein lysates were extracted from frozen tissue using the radioimmunoprecipitation (RIPA) buffer containing 'Complete', which is a protease inhibitor cocktail (Roche, Penzberg, Germany). The Pierce BCA protein assay reagent kit (Pierce Biotechnology, Inc., Rockford, IL, USA) was applied for quantification of total protein.

Sandwich-type enzyme-linked immunosorbent assay. CCL20 protein levels in the different tissue lysates were determined by sandwich-type enzyme-linked immunosorbent assays (ELISA) according to the manufacturer's protocol. Duplicates were assayed for all samples, and the protein levels were calculated as the mean of two measurements. A validated commercial ELISA kit (DuoSet DY360; R\&D Systems, Minneapolis, MN, USA) was used to determine the protein levels of CCL20 (SwissProt, P78556). The absorbance was analyzed at a wavelength of $450 \mathrm{~nm}$ (530 nm reference wavelength) in a 96-well microtiter plate reader (Unimax 2010; Heidolph Instruments, Schwabach, Germany). CCL20 protein concentration from each cell lysate was normalized to the total protein content of each sample.

Immunohistochemistry. Surgically resected specimens were fixed in formalin, embedded in paraffin, cut into $4-\mu \mathrm{m}$ sections and mounted on Superfrost Plus slides (Thermo Fisher Scientific, Waltham, MA, USA). After deparaffinization and rehydration from graded ethanol to deionized water, the sections were microwaved in target retrieval solution pH 9.0 (Target Retrieval; Dakocytomation, Carpinteria, CA, USA). Endogeneous peroxidase activity was blocked with $3 \%$ hydrogen peroxide. Further blocking steps were performed with normal rabbit serum, avidin and biotin (Vector Laboratories, Burlingame, CA, USA). Overnight incubation of slides with primary goat polyclonal anti-human CCL20 antibody (15 $\mu \mathrm{g} / \mathrm{ml}$, AF360; R\&D Systems) was followed by incubation with secondary biotinylated rabbit anti-goat IgG antibody and the avidin-biotin-peroxidase reaction (Vector Laboratories). After color reaction with aminoethylcarbazide solution (Merck KGaA, Darmstadt, Germany) tissue sections were counterstained with haematoxylin and mounted with Aquatex. Specimens incubated without primary antibody served as the negative control.
In situ hybridization. For the detection of miR-21, the miRCURY LNA ${ }^{\mathrm{TM}}$ microRNA ISH optimization kit (FFPE) (Exiqon A/S, Vedbaek, Denmark) was applied. The specimens were fixed in formalin, embedded in paraffin, cut in $6-\mu \mathrm{m}$ sections and mounted on Superfrost Plus slides. The sections were deparaffinized and rehydrated from graded ethanol to deionized water. Subsequently, specimens were incubated for $24 \mathrm{~min}$ with proteinase $\mathrm{K}(15 \mu \mathrm{g} / \mathrm{ml}$; Exiqon A/S $)$. After dehydration, $150 \mathrm{nM}$ of the specific probe was added to each specimen in hybridization buffer (Exiqon A/S). A probe complementary to U6 snRNA was applied as a positive control, and a scrambled probe not known to be complementary to any endogeneous miRNA was used as a negative control (Exiqon A/S). U6 snRNA is a small nucleic acid present in abundance in the nucleus of all cells and is therefore easy to detect.

Subsequently, a coverslip was attached, sealed with Fixogum and slides were incubated for $1 \mathrm{~h}$ at $57^{\circ} \mathrm{C}$. Consequently, coverslips were removed and sections were washed in decreasing concentrations of SSC buffer (Life Technologies) at the hybridization temperature. Endogenous alkaline phosphatase was blocked with blocking solution (Roche Applied Science, Indianapolis, IN, USA) for $15 \mathrm{~min}$ followed by incubation with an anti-DIG antibody for $60 \mathrm{~min}$ (Roche Applied Science). The color reaction with AP substrate (NBT/BCIP tablet; Roche Applied Science) was stopped with the KTBT buffer after $1 \mathrm{~h}$. After counterstaining with Nuclear Fast Red (Sigma-Aldrich, St. Louis, MO, USA) for $1 \mathrm{~min}$, the sections were dehydrated and mounted with Eukitt (Sigma-Aldrich).

Calculations and statistical methods. CCL20 and miRNA expression profiles of the different groups are shown as the means and standard error of the means (SEM). Where appropriate, the Student's t-test was applied to test for group differences of continuous variables. All calculations were carried out using SPSS, version 19. The significance level was $\mathrm{P}<0.05$.

\section{Results}

CCL20 mRNA and protein expression in CRC tissues. To study CCL20 gene expression at the mRNA and protein levels, tissue samples from patients with different clinicopathological features of CRC were analyzed using q-RT-PCR and ELISA. Since the regulated control of target genes by miRNAs may be achieved by degradation of their mRNA, we monitored changes in CCL20 mRNA expression by q-RT-PCR in 46 CRC tissue samples in relation to the tumor-free corresponding tissues as reference. Independent of the patient charateristics, CCL20 mRNA expression was significantly increased in the tumor tissues $(\mathrm{P}<0.05)$ when compared to expression in the normal mucosa (Fig. 1). As regulated control of target genes by miRNAs may also be achieved by inhibition of translation, we subsequently monitored changes in CCL20 protein expression in 46 CRC tissue samples from the same patient cohort applying an ELISA assay specific for CCL20. In line with the CCL20 mRNA expression data, CCL20 protein expression was significantly increased in the tumor tissues $(\mathrm{P}<0.05)$ independently of T-classification and differentiation or clinical stage (Fig. 2). 

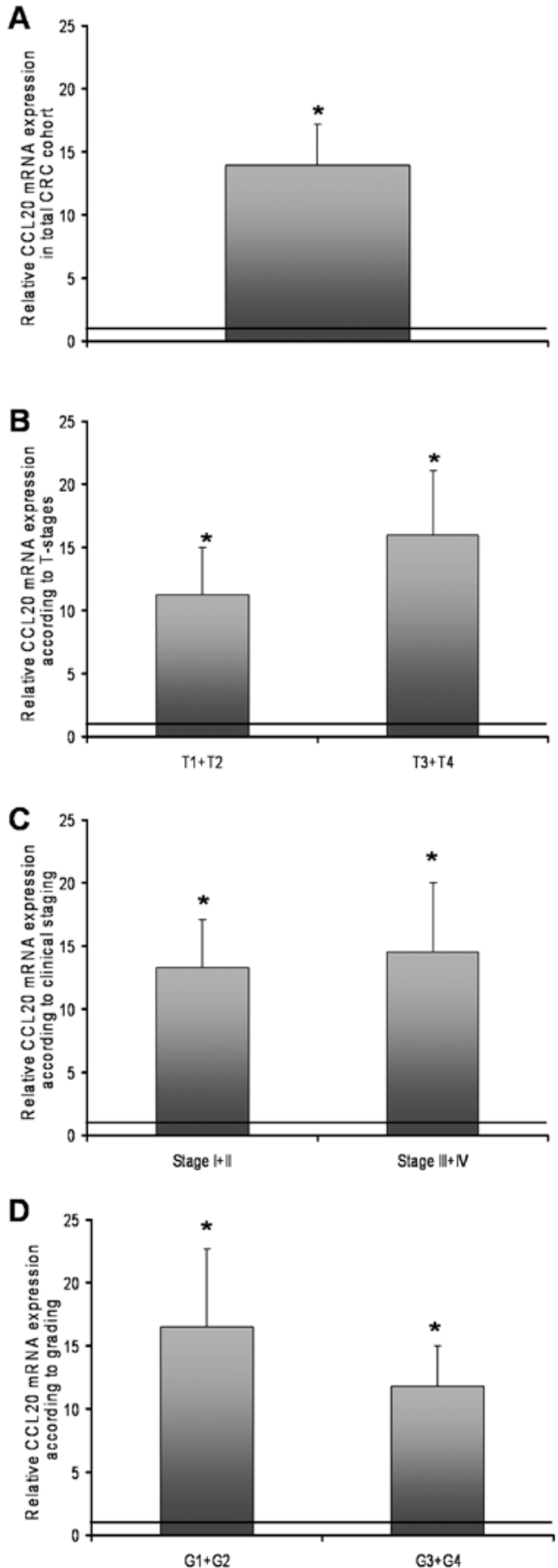

Figure 1. CCL20 mRNA expression in CRC of different clinical characteristics as determined by q-RT-PCR. q-RT-PCR data are expressed as means \pm standard error of the mean (SEM), ${ }^{*} \mathrm{P}<0.05, \mathrm{n}=46$. Fold increase $>1$ indicates CCL20 overexpression in CRC tissues relative to the unaffected adjacent tissues, respectively. (A) Mean data of all investigated patients. (B) Mean patient data according to T-classification. (C) Mean patient data according to clinical staging. (D) Mean patient data according to differentiation states.

miR-21 expression in the CRC tissues. Analyzing CCL20 mRNA and protein expression in CRC tissues revealed significant overexpression. Subsequently, we determined miR-21 expression in the same $46 \mathrm{CRC}$ tissue samples compared to the corresponding normal tissues applying special miRNA expression assays for q-RT-PCR. Based on our functional tests, we were expecting an antidromic expression pattern of

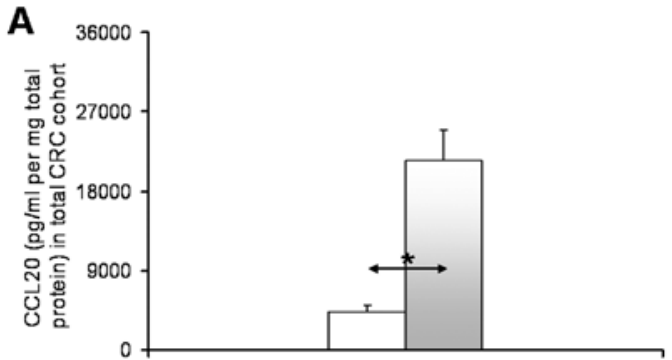

$\square$ corresponding tumor free tissues $\square$ CRC tissues

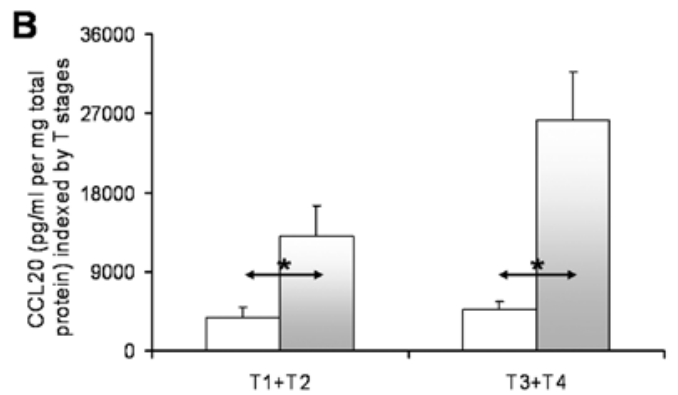

$\square$ corresponding tumor free tissues $\quad$ QCRC tissues
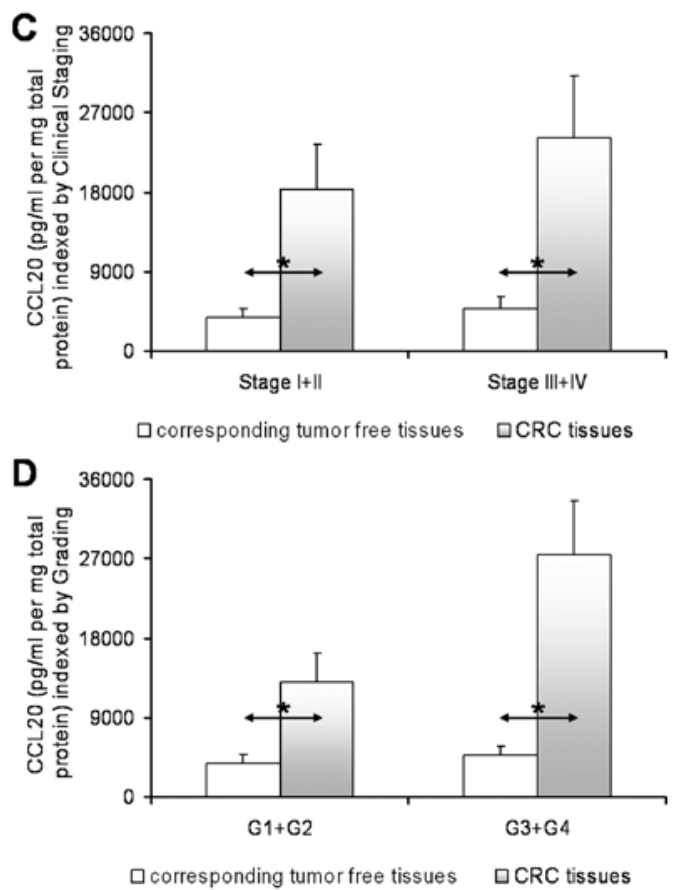

Figure 2. CCL20 protein expression in CRC of different clinical characteristics as determined by ELISA assays. ELISA results are presented as absolute values of $\mathrm{pg} / \mathrm{ml}$ chemokine ligand per $\mathrm{mg}$ total protein in CRC tissues and unaffected adjacent tissues, respectively. The data are expressed as means \pm standard error of the mean (SEM), ${ }^{*} \mathrm{P}<0.05, \mathrm{n}=46$. (A) Mean data of all investigated patients. (B) Mean patient data according to T-classification. (C) Mean patient data according to clinical staging. (D) Mean patient data according to differentiation states.

miR-21 and CCL20 provided both molecules are expressed in the same cell. Yet, in the total CRC patient cohort, the mean miR-21 expression was significantly $(\mathrm{P}<0.05)$ increased in tumor samples relative to corresponding non-affected tissues (Fig. 3A). However, analysis of the clinicopathological features of the total patient cohort revealed that significantly increased miR-21 expression was restricted only to advanced 
A
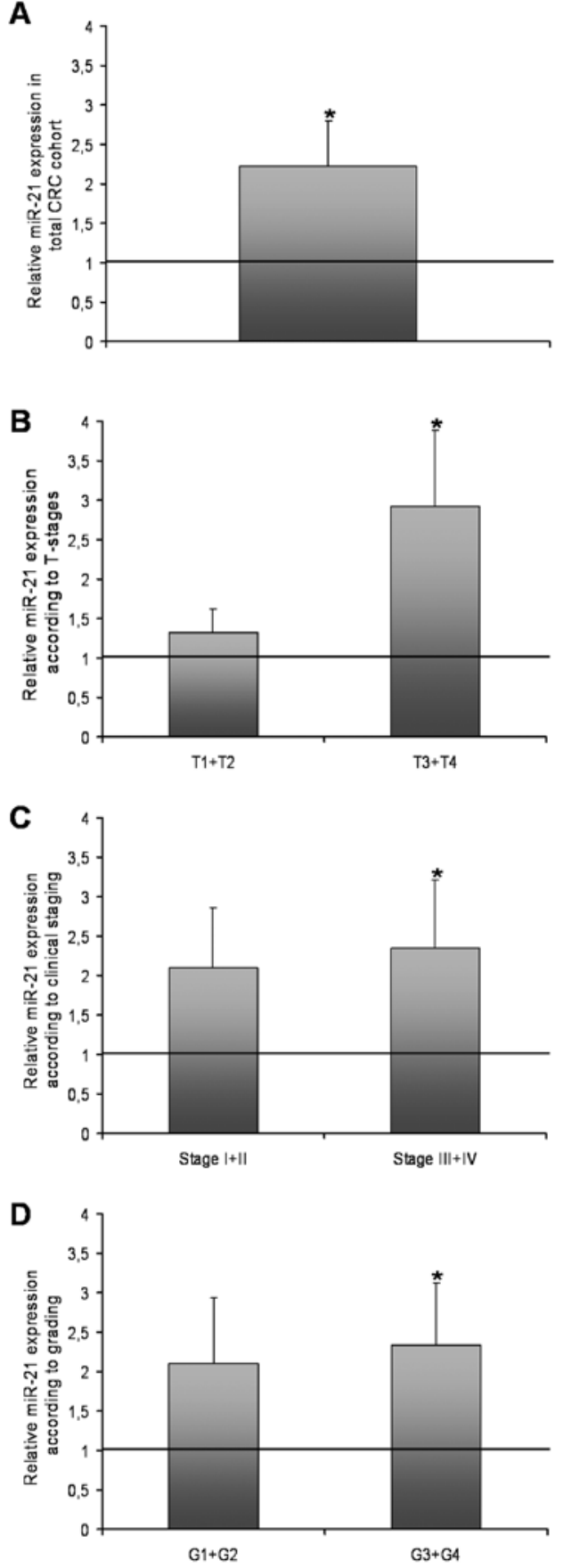

Figure 3. miR-21 expression in CRC of different clinical characteristics as determined by q-RT-PCR. q-RT-PCR data are expressed as means \pm standard error of the mean (SEM), ${ }^{*} \mathrm{P}<0.05, \mathrm{n}=46$. Fold increase $>1$ indicates miR-21 overexpression in CRC tissues relative to unaffected adjacent tissues, respectively. (A) Mean data of all investigated patients. (B) Mean patient data according to T-classification. (C) Mean patient data according to clinical staging. (D) Mean patient data according to differentiation states.

$\mathrm{T}, \mathrm{G}$ and clinical stages (T3+T4 and $\mathrm{G} 3+\mathrm{G} 4, \mathrm{III}+\mathrm{IV})$ $(\mathrm{P}<0.05)$ (Fig. 3B-D).

In contrast to our expectations, CRC tissue expression analysis revealed that CCL20 and miR-21 were not inversely expressed in CRC tissue samples but were both shown to be significantly upregulated (Figs. 1A, 2A and 3A).

Monitoring CCL20/miR-21 cellular localization in CRC tumor sections. The fact that no antidromic expression pattern
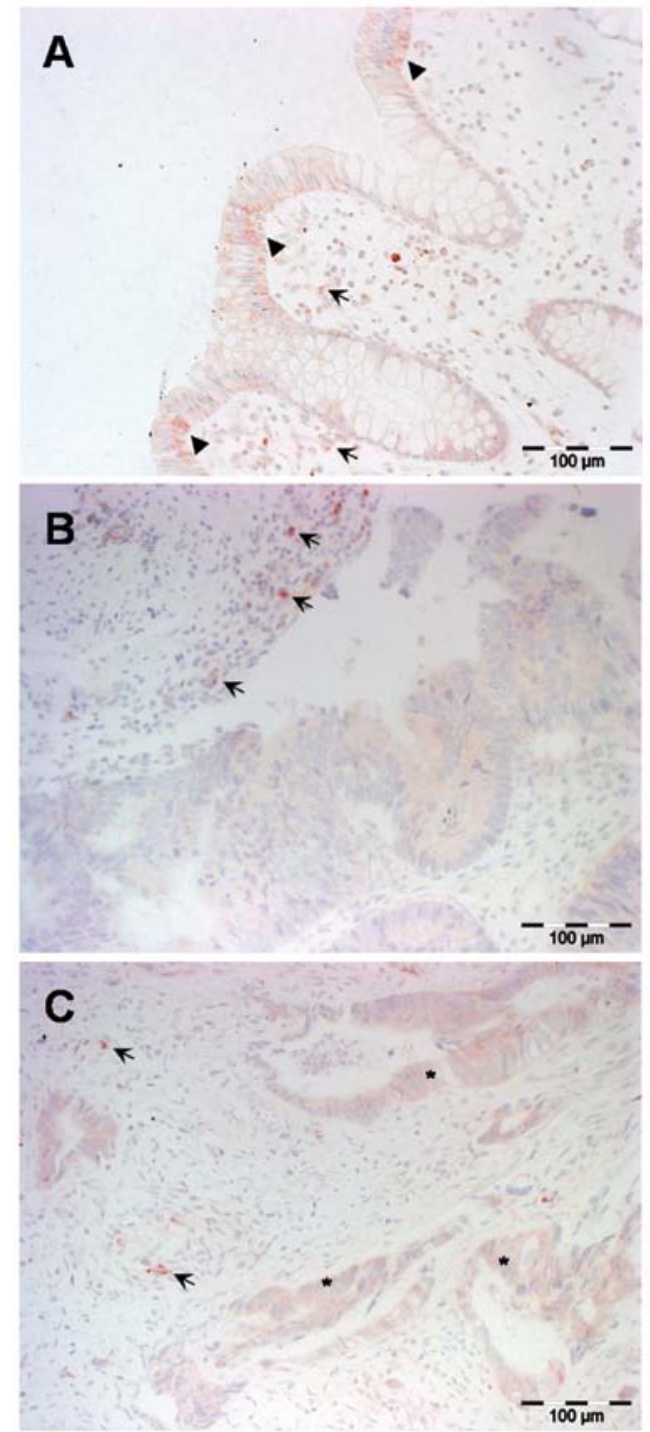

Figure 4. Localization of CCL20 protein expression in CRC. Representative specimens of the normal mucosa as well as of the colorectal adenocarcinomas were probed with antibodies against CCL20 (15 $\mu \mathrm{g} / \mathrm{ml}$; ABC method). (A) In unaffected colorectal tissues, immunoreactivity against CCL20 was detected in the perinuclear regions of mucosal epithelial cells (arrowheads) and mesenchymal cells (arrows). (B) In the colorectal tissue specimens, immunoreactive signals against CCL20 were predominantly detected in the mesenchymal portions (arrows) while no anti-CCL20 signals were observed in adenocarcinoma cells. (C) One specimen revealed immunoreactivity against CCL20 in both adenocarcinoma cells (black asterisks) and mesenchymal elements (black arrows).

was noted in CRC tissues indicated that miR-21 and CCL20 may not be expressed in the same cell. To test this hypothesis, we investigated the cellular localization of miR-21 and its target gene CCL20 in 8 formalin-fixed paraffin-embedded CRC tissue sections using an immunohistochemical approach and in situ hybridization, respectively.

Monitoring CCL2O cellular localization in CRC tumor sections. Based on an immunohistochemical approach, we investigated the cellular distribution of CCL20 in 8 formalin-fixed paraffin-embedded CRC tissue sections using tumor-free corresponding colonic tissues as reference. In adjacent normal tissues, CCL20 expression was observed predominantly in the perinuclear regions of mucosal epithelial 


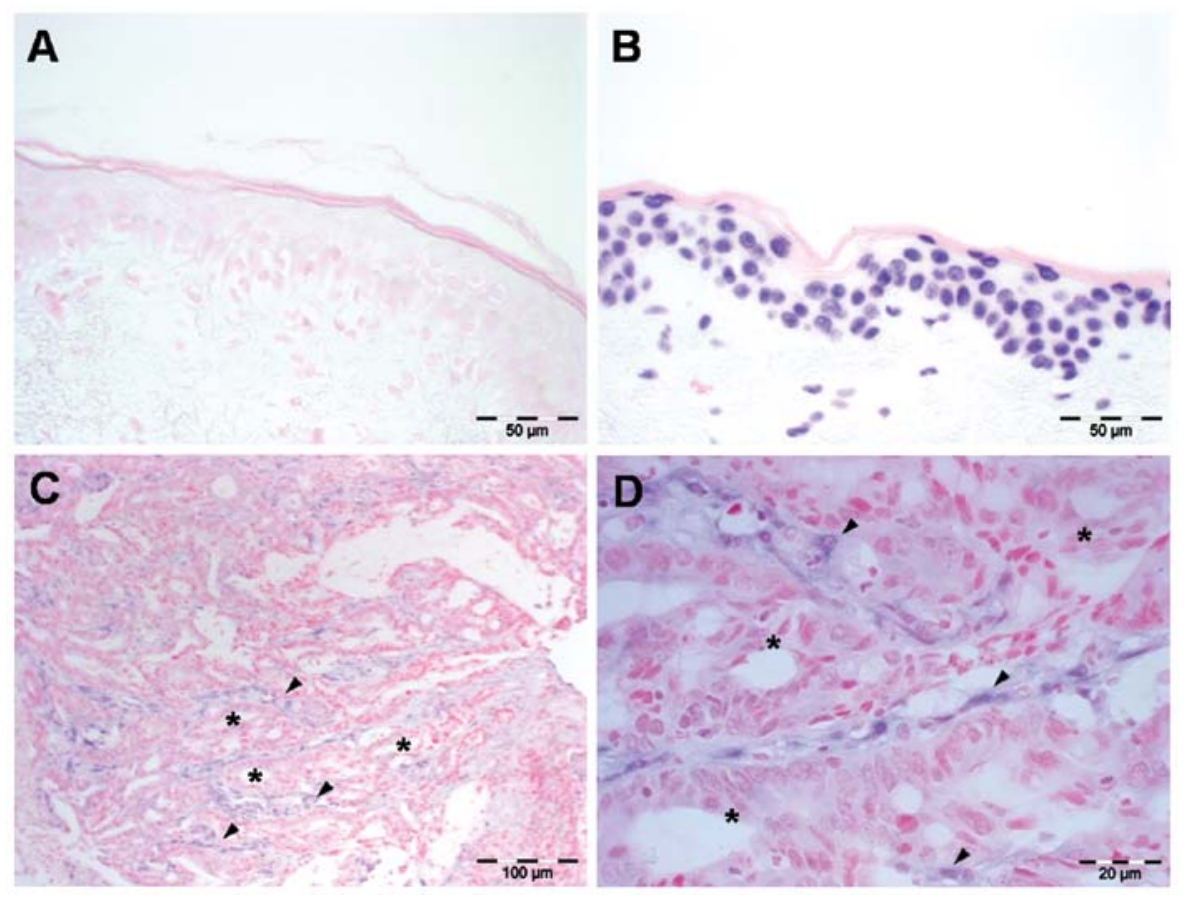

Figure 5. Localization of miR-21 expression in CRC. Representative specimens of colorectal adenocarcinomas were hybridized with probes against miR-21 ( $150 \mathrm{nM}$, alkaline phosphatase method, counterstained with Nuclear Fast Red). As positive control, a probe complementary to U6 snRNA (1 nM) was applied and a scrambled probe $(25 \mathrm{nM})$ not known to be complementary to any endogeneous miRNA served as a negative control. (A) Applying a negative control resulted in no detectable signal while (B) in situ hybridization with the probe complementary to U6 snRNA showed positive reactivity in the majority of nuclei without substantial background reactivity. (C and D) Positive reactivity against miR-21 was predominantly detected in the cytoplasmic compartments of tumorassociated fibroblasts and to a minor degree in immune cells such as macrophages and lymphocytes. Arrowheads indicate mesenchymal areas with positive miR-21-associated signaling in the mircroenvironment of the tumor. Asterisks indicate tumor areas negative for miR-21 staining. (C) Lower magnification. (D) Detailed magnification.

cells (Fig. 4A). In addition, mesenchymal cells also exhibited positive CCL20 signals (Fig. 4A). While CCL20 expression profiling in CRC tissues indicated significant upregulation, the cellular distribution of CCL20 revealed that the tumor areas were negative for CCL20 reactivity. Positive CCL20-associated signaling was almost exclusively observed in mesenchymal elements such as macrophages or lymphocytes in the tumor microenvironment (Fig. 4B). Thus, only 1 out of 8 CRC tissue sections under investigation showed positive CCL20 signals which were attributed to adenocarcinoma cells as well as to mesenchymal elements exhibiting positive reactivity against CCL20 (Fig. 4C). Hence, the vast majority of CCL20 expression found in CRC tissues was restricted to the mesenchymal elements. Therefore, it may be concluded that the significantly upregulated CCL20 expression exhibited by the majority of CRC patients under investigation was related to an increase in mesenchymal cells associated with infiltration of immune cells such as macrophages or lymphocytes to the site of the tumor.

Monitoring miR-21 cellular localization in CRC tumor sections. Next, we examined the cellular localization of miR-21 in the same 8 formalin-fixed paraffin embedded CRC tissue samples by situ hybridization experiments. While the negative control resulted in no detectable signal (Fig. 5A), in situ hybridization with the probe complementary to U6 snRNA showed positive reactivity in the majority of nuclei without substantial background reactivity (Fig. 5B). In situ hybridization experiments with miR-21 probes resulted in positive reactivity exclusively restricted to mesenchymal areas in the mircroenvironment of CRC tumor tissues (Fig. 5C and D). Hence, tumor-associated fibroblasts were shown to exhibit considerable miR-21 reactivity in their cytoplasmic compartments. To a minor degree, positive miR-21-associated signaling was also detected in immune cells such as macrophages and lymphocytes which were recruited to the site of the tumor. However, no positive miR-21 reactivity was observed in tumor cells of CRC patients (Fig. 5C and D). Thus, the significant miR-21 overexpression detected in the majority of CRC patients under investigation can now be allocated to the vast number of tumor-associated fibroblasts and to an increase in mesenchymal cells associated with the infiltration of immune cells whereas tumor cells may be excluded as the source of miR-21 expression in CRC tissues.

\section{Discussion}

In a recent study (16), we demonstrated a functional interaction between miR-21 and CCL20 in various CRC cell lines suggesting the regulation of CCL20 expression may be orchestrated under the control of miR-21. In the present study we explored whether such functional interactions are permitted at the cellular level which requires co-expression of miR-21 and CCL20 in the same cell. Likewise, we would expect an inverse correlation of expression of the two molecules in CRC tissue samples. However, our first set of experiments investigating the expression profile of CCL20/miR-21 in tissue samples of CRC patients revealed that CCL20 and miR-21 were both significantly upregulated in CRC tissues. Thus, we 
observed significant CCL20 gene and protein overexpression independent of clinicopathological patient characteristics such as T-classification and differentiation or clinical staging.

In contrast, clinicopathological differentiation of CRC patients with respect to miR-21 expression revealed significant upregulation only in late $\mathrm{T}, \mathrm{G}$ and clinical stages (T3+T4, G1+G2 and stages III and IV). These data correlate with a recent study (23) showing a significant trend of higher miR-21 levels with progression of CRC although these results only refer to patients that did not develop metastases compared to patients that developed metastases. While Vickers et al (23) demonstrated significant miR-21 overexpression in both patient groups under investigation, we observed significant miR-21 upregulation exclusively in patients with more advanced or less differentiated tumors. Likewise, another study associated miR-21 expression with the development of distant metastases and clinical stages of CRC and also with lymph node positivity (24). Moreover, the authors demonstrated increased miR-21 levels from well (G1) to poorly (G3) differentiated tumors. These data are in compliance with our results indicating an association of higher miR-21 expression with a decreased differentiation state of CRC tissues. Thus, miR-21 expression may be linked to clinicopathological patient characteristics and progression of the tumors under investigation.

However, with respect to significantly upregulated CCL20 and miR-21 expression in CRC tissues, our results were contrary to our expectations. We expected an inverse expression pattern in CRC tissues since we had previously shown that miR-21 interacts with the 3' UTR of CCL20 mRNA in CRC cell lines. Showing no antidromic expression pattern in CRC tissues provided a first clue that miR-21 and CCL20 may not be expressed in the same cell. Hence, investigation of their cellular localization revealed that miR-21 is predominantly expressed in mesenchymal compartments particularly in tumor-assocated fibroblasts and in some infiltrating immune cells, e.g. macrophages of the tumor but not within tumor cells. These findings correlate with other studies describing miR-21 expression primarily in tumor-associated fibroblasts in the stroma of CRC tumors $(25,26)$. Similarly, miR-21 expression was localized to the stroma rather than to the cancer areas in other cancer entities including breast cancer (27) and pancreatic cancer (28). Therefore, our results demonstrated that miR-21 overexpression in CRC tissues is not associated with tumor cells. Hence, it may be speculated that miR-21 expression in tumor-associated fibroblasts is induced by paracrine signaling of CRC cells or infiltrating immune cells. It is well established that carcinogenesis is a process involving the attraction of immune cells to the tumor microenvironment $(29,30)$. Since miR-21 is predominantly expressed by fibroblasts but also by immune cells such as macrophages and lymphocytes it may be concluded that the significant miR-21 upregulation observed in CRC tissues is in part also supported by the tumor-conducted attraction of immune cells. Likewise, the significant upregulation of CCL20 mRNA and protein we observed in CRC tissues may be explained by the tumor-conducted attraction of immune cells to the tumor microenvironment. This conclusion is in line with recent studies suggesting that upregulation of CCL20 in CRC and adenomas may be due to the inflammatory microenvironment accompanying both the benign and malignant neoplasms (31).
Moreover, applying semi-quantitative immunohistochemistry, Brand et al (32) observed in 9 of 15 CRCs under investigation that CCL20 expression was lost or decreased in comparison to normal colonic tissue suggesting that CCL20 is downregulated in cancerous and metastatic tissues. These results are also concordant with our immunohistochemistry-based findings showing that CCL20 expression is predominantly located in mesenchymal elements in the tumor microenvironment including infiltrating immune cells. In contrast, almost all tumors under investigation turned out to be negative for CCL20 staining. Our observations are further supported by recent investigations in a mouse model where also CRC tumorassociated macrophages expressed CCL20, thereby recruiting CCR6-positive regulatory $\mathrm{T}$ cells leading to tumor progression (30). Moreover, our results showed that the increased CCL20 expression we observed in CRC tissues is essentially due to the infiltration of immune cells which secrete CCL20 thereby influencing the behavior of CRC cells. In this context, the cellular composition of the tissue samples under investigation represents a critical factor determining the outcome of an expression profiling experiment. With respect to CCL20, the portion of infiltrating immune cells in the investigated tumor samples may predominantly account for the increased CCL20 expression; whereas for miR-21 expression the portion of tumor-associated fibroblasts may primarily account for the miR-21 overexpression detected in the tumor samples. Hence, variations in the composition of the different cell types in tumor samples may explain the high variability of expression we observe between individual patients.

It may also be speculated that CCL20 secreted by immune cells influences miR-21 expression in tumor-associated fibroblasts via CCR6 signaling. Altered miRNA expression upon chemokine stimulation has recently been shown for the CXCL12/CXCR4 axis in breast cancer (33).

Although we previously demonstrated in vitro that miR-21 downregulates CCL20 expression in CRC cell lines and functionally interacts with the 3'-UTR of CCL20 (16), we showed here that miR-21 and CCL20 are expressed predominantly in different cell types in the microenvironment of colorectal tumors. Thus, our results clearly exclude the chance of miR-21 and CCL20 being expressed in the same cell. Thus, a functional interaction between miR-21 and CCL20 in vivo is rather unlikely.

In conclusion, our results revealed that the cellular composition of tissues and the degree of immune cell infiltration constitute a critical factor determining the outcome of an expression profiling experiment. Moreover, it became evident that due to their locally separated expression in different cell types in the microenvironment of colorectal tumors, regulatory in vivo interactions between miR-21 and CCL20 constitute an unlikely option. To enable a functional interaction in vivo, miRNA and the target need to be expressed in the same cell. Consequently, the pure knowledge of dysregulated expression of miRNAs and their target genes in tissues does not allow an assumption on regulatory interactions as long as their accurate localization is not known.

\section{Acknowledgements}

We thank B. Kruse for the excellent technical assistance. 


\section{References}

1. Bartel DP: MicroRNAs: genomics, biogenesis, mechanism, and function. Cell 116: 281-297, 2004.

2. Brennecke J, Stark A, Russell RB and Cohen SM: Principles of microRNA-target recognition. PLoS Biol 3: e85, 2005.

3. Valencia-Sanchez MA, Liu J, Hannon GJ and Parker R: Control of translation and mRNA degradation by miRNAs and siRNAs Genes Dev 20: 515-524, 2006.

4. Lewis BP, Burge CB and Bartel DP: Conserved seed pairing, often flanked by adenosines, indicates that thousands of human genes are microRNA targets. Cell 120: 15-20, 2005.

5. Lee Y, Kim M, Han J, Yeom KH, Lee S, Baek SH and Kim VN: MicroRNA genes are transcribed by RNA polymerase II EMBO J 23: 4051-4060, 2004

6. Borchert GM, Lanier W and Davidson BL: RNA polymerase III transcribes human microRNAs. Nat Struct Mol Biol 13: 1097-1101, 2006.

7. Han J, Lee Y, Yeom KH, Kim YK, Jin H and Kim VN: The Drosha-DGCR8 complex in primary microRNA processing. Genes Dev 18: 3016-3027, 2004

8. Wang X, Xu X, Ma Z, Huo Y, Xiao Z, Li Y and Wang Y: Dynamic mechanisms for pre-miRNA binding and export by Exportin-5. RNA 17: 1511-1528, 2011.

9. Mocellin S, Pasquali S and Pilati P: Oncomirs: from tumor biology to molecularly targeted anticancer strategies. Mini Rev Med Chem 9: 70-80, 2009.

10. Tsuruta T, Kozaki K, Uesugi A, Furuta M, Hirasawa A, Imoto I, Susumu N, Aoki D and Inazawa J: miR-152 is a tumor suppressor microRNA that is silenced by DNA hypermethylation in endometrial cancer. Cancer Res 71: 6450-6462, 2011.

11. Yan LX, Wu QN, Zhang Y, Li YY, Liao DZ, Hou JH, Fu J, Zeng MS, Yun JP, Wu QL, Zeng YX and Shao JY: Knockdown of miR-21 in human breast cancer cell lines inhibits proliferation, in vitro migration and in vivo tumor growth. Breast Cancer Res 13: R2, 2011.

12. Chang KH, Miller N, Kheirelseid EA, Lemetre C, Ball GR, Smith MJ, Regan M, McAnena OJ and Kerin MJ: MicroRNA signature analysis in colorectal cancer: identification of expression profiles in stage II tumors associated with aggressive disease. Int J Colorectal Dis 26: 1415-1422, 2011.

13. Luo X, Burwinkel B, Tao S and Brenner H: MicroRNA signatures: novel biomarker for colorectal cancer? Cancer Epidemiol Biomarkers Prev 20: 1272-1286, 2011.

14. Fassan M, Pizzi M, Giacomelli L, Mescoli C, Ludwig K, Pucciarelli S and Rugge M: PDCD4 nuclear loss inversely correlates with miR-21 levels in colon carcinogenesis. Virchows Arch 458: 413-419, 2011.

15. Hu N, Zhang J, Cui W, Kong G, Zhang S, Yue L, Bai X, Zhang Z, Zhang $\mathrm{W}$, Zhang $\mathrm{X}$ and $\mathrm{Ye} \mathrm{L}$ : miR-520b regulates migration of breast cancer cells by targeting hepatitis B X-interacting protein and interleukin-8. J Biol Chem 286: 13714-13722, 2011.

16. Vicinus B, Rubie C, Faust SK, Frick VO, Ghadjar P, Wagner M, Graeber S and Schilling MK: miR-21 functionally interacts with the 3'UTR of chemokine CCL20 and down-regulates CCL20 expression in miR-21 transfected colorectal cancer cells. Cancer Lett 316: 105-112, 2012

17. Rubie C, Oliveira V, Kempf K, Wagner M, Tilton B, Rau B, Kruse B, Konig J and Schilling M: Involvement of chemokine receptor CCR6 in colorectal cancer metastasis. Tumour Biol 27: 166-174, 2006.

18. Ghadjar P, Coupland SE, Na IK, Noutsias M, Letsch A, Stroux A, Bauer S, Buhr HJ, Thiel E, Scheibenbogen C and Keilholz U: Chemokine receptor CCR6 expression level and liver metastases in colorectal cancer. J Clin Oncol 24: 1910-1916, 2006.

19. Du ZM, Hu LF, Wang HY, Yan LX, Zeng YX, Shao JY and Ernberg I: Upregulation of miR-155 in nasopharyngeal carcinoma is partly driven by LMP1 and LMP2A and downregulates a negative prognostic marker JMJD1A. PLoS One 6: e19137, 2011.
20. Aprelikova O, Yu X, Palla J, Wei BR, John S, Yi M, Stephens R, Simpson RM, Risinger JI, Jazaeri A and Niederhuber J: The role of miR-31 and its target gene SATB2 in cancer-associated fibroblasts. Cell Cycle 9: 4387-4398, 2010.

21. Bustin SA: Absolute quantification of mRNA using real-time reverse transcription polymerase chain reaction assays. J Mol Endocrinol 25: 169-193, 2000.

22. Rubie C, Kempf K, Hans J, Su T, Tilton B, Georg T, Brittner B, Ludwig B and Schilling M: Housekeeping gene variability in normal and cancerous colorectal, pancreatic, esophageal, gastric and hepatic tissues. Mol Cell Probes 19: 101-109, 2005.

23. Vickers MM, Bar J, Gorn-Hondermann I, Yarom N, Daneshmand M, Hanson JE, Addison CL, Asmis TR, Jonker DJ, Maroun J, Lorimer IA, Goss GD and Dimitroulakos J: Stage-dependent differential expression of microRNAs in colorectal cancer: potential role as markers of metastatic disease. Clin Exp Metastasis 29: 123-132, 2012.

24. Slaby O, Svoboda M, Fabian P, Smerdova T, Knoflickova D, Bednarikova M, Nenutil R and Vyzula R: Altered expression of miR-21, miR-31, miR-143 and miR-145 is related to clinicopathologic features of colorectal cancer. Oncology 72: 397-402, 2007.

25. Nielsen BS, Jørgensen S, Fog JU, Søkilde R, Christensen IJ, Hansen U, Brünner N, Baker A, Møller S and Nielsen HJ: High levels of microRNA-21 in the stroma of colorectal cancers predict short disease-free survival in stage II colon cancer patients. Clin Exp Metastasis 28: 27-38, 2011.

26. Yamamichi N, Shimomura R, Inada K, Sakurai K, Haraguchi T, Ozaki Y, Fujita S, Mizutani T, Furukawa C, Fujishiro M, Ichinose M, Shiogama K, Tsutsumi Y, Omata M and Iba H: Locked nucleic acid in situ hybridization analysis of miR-21 expression during colorectal cancer development. Clin Cancer Res 15: 4009-4016, 2009.

27. Rask L, Balslev E, Jørgensen S, Eriksen J, Flyger H, Møller S, Høgdall E, Litman T and Nielsen BS: High expression of miR-21 in tumor stroma correlates with increased cancer cell proliferation in human breast cancer. APMIS 119: 663-673, 2011.

28. du Rieu MC, Torrisani J, Selves J, Al Saati T, Souque A, Dufresne M, Tsongalis GJ, Suriawinata AA, Carrère N, Buscail L and Cordelier P: MicroRNA-21 is induced early in pancreatic ductal adenocarcinoma precursor lesions. Clin Chem 56: 603-612, 2010.

29. Bossard C, Bézieau S, Matysiak-Budnik T, Volteau C, Laboisse CL, Jotereau F and Mosnier JF: HLA-E/ $\beta 2$ microglobulin overexpression in colorectal cancer is associated with recruitment of inhibitory immune cells and tumor progression. Int J Cancer 131: 855-863, 2012.

30. Liu J, Zhang N, Li Q, Zhang W, Ke F, Leng Q, Wang H, Chen J and Wang H: Tumor-associated macrophages recruit CCR6 ${ }^{+}$ regulatory $\mathrm{T}$ cells and promote the development of colorectal cancer via enhancing CCL20 production in mice. PLoS One 6: e19495, 2011.

31. McLean MH, Murray GI, Stewart KN, Norrie G, Mayer C, Hold GL, Thomson J, Fyfe N, Hope M, Mowat NA, Drew JE and El-Omar EM: The inflammatory microenvironment in colorectal neoplasia. PLoS One 6: e15366, 2011.

32. Brand S, Olszak T, Beigel F, Diebold J, Otte JM, Eichhorst ST, Göke B and Dambacher J: Cell differentiation dependent expressed CCR6 mediates ERK-1/2, SAPK/JNK, and Akt signaling resulting in proliferation and migration of colorectal cancer cells. J Cell Biochem 97: 709-723, 2006.

33. Rhodes LV, Bratton MR, Zhu Y, Tilghman SL, Muir SE, Salvo VA, Tate CR, Elliott S, Nephew KP, Collins-Burow BM and Burow ME: Effects of SDF-1-CXCR4 signaling on microRNA expression and tumorigenesis in estrogen receptor-alpha (ER- $\alpha$ )-positive breast cancer cells. Exp Cell Res 317: 2573-2581, 2011. 